\title{
Analytical studies of Gibbs-Thomson effect on the diffusion controlled spherical phase growth in a subcooled medium
}

\author{
T. Wu, Y.-Z. Chen
}

\begin{abstract}
By using a small-time series expansion technique, the thermal effect of surface tension (GibbsThomson effect) on the early-stage phase growth of a spherical nucleus immersed in an infinite subcooled liquid is studied in this paper. The result shows that surface tension greatly reduces the incipient growth rate of the solid nucleus. Critical value of surface tension is found beyond which the decreasing of the phase growth rate with time becomes non-monotonic. Analytical expression for the phase growth rate in terms of relevant physical parameters is also derived under the condition of small degree of undercooling.
\end{abstract}

Keywords Gibbs-Thomson effect, Surface tension, Spherical phase growth, Solidification

\section{1}

\section{Introduction}

Growth of a solid nucleus immersed in an infinite subcooled liquid initiates a motion of an interface between two media. The solidification process is normally modeled by a set of diffusion equations, and the advancement of the interface (freezing front) is controlled by the diffusion of the latent heat, which is released from the freezing front in the course of a phase transformation, into the surrounding media. The phenomenon is found in many familiar physical processes involving phase changes in their development. Traditionally, these events are categorized and well documented under the name 'Stefan problem' [1-2]. The simplest theoretical treatment of the phase growth problem assumes the solid nucleus to be spherical in shape. Without considering the surface tension at the interface, it has been shown that the diffusion equation allows a self-similar solution with spherical symmetry [3], and the growth of the radius of the solid sphere $R^{*}$ was found to be proportional to the square root of the elapsing time $t^{*}$. It is well established that the solid sphere cannot stably maintain its radial symmetry for long (Mullins-

Received: 4 October 2001

Published online: 4 September 2002

(C) Springer-Verlag 2002

T. Wu ( $\varangle)$, Y.-Z. Chen

Department of Mechanical Engineering,

National Taiwan University, Taipei 106, Taiwan

E-mail: tywu@ccms.ntu.edu.tw

Tel.: +886-2-23630231 ext 2413 ext 21

Fax: +886-2-23631755
Sekerka instability [4]). Once the instability sets in, the solid phase starts to evolve into, preferably, a needle shape and subsequently branches out to form a dendrite-like structure [5-7]. Recent investigations [8-10] have revealed that the surface tension plays a decisive role in the mode selection mechanism during the complex dendritic pattern formation. Whereas more fundamentally, as will be shown in this paper, the surface tension also has a significant effect on the spherical growth of the nucleus during its early-stage development.

Surface tension affects the equilibrium temperature (freezing or fusion temperature) at the interface of the two phases via the so-called Gibbs-Thomson law. If we let $\gamma$ denote the surface tension coefficient, $L$ the latent heat per unit volume of the solid phase, $R^{*}$ the radius of the solid sphere, and $T_{f}^{*}$ the freezing/fusion temperature on a planar interface; then the equilibrium temperature at the spherical solid/liquid interface is given by the following Gibbs-Thomson condition [11, 12]:

$T^{*}=T_{f}^{*}\left(1-\frac{2 \gamma}{L R^{*}}\right)$

The Gibbs-Thomson law predicts a reduction of the freezing temperature on a curved interface, and the amount of difference vanishes with increasing radius of curvature. Since the temperature of the liquid must be lower than that given in (1) for a possible solidification to occur; thus for a given temperature $T_{\infty}^{*}$ of the subcooled liquid, there exists a limiting size of the nucleus

$R_{0}^{*}=2 \gamma T_{f}^{*} / L\left(T_{f}^{*}-T_{\infty}^{*}\right)$

below which crystallization cannot proceed. In the present study, we shall assume that initially there exists already in the medium a solid nucleus whose size is greater than the limiting value, and focus our attention primarily on its subsequent growth under the modified equilibrium condition (1) as imposed by the surface tension at the interface.

Formulation of the present problem is based on the classical one-phase model [1-2] that has been traditionally applied to most of the Stefan problems. In this one-sided approach, the latent heat released from the freezing front is assumed to be carried away by the surrounding liquid only. The assumption is a legitimate one as long as the immersed solid sphere remains small. Furthermore, the small convective motion of fluid induced by the slight density change in the phase transformation is of higher order in magnitude and hence can be neglected from the 
heat equation. The simplification leads to the familiar unsteady heat-diffusion equation for the liquid phase with moving interfacial boundary. The equation governing the progression of the interface is derived from equating the amount of heat released per increase of the freezing layer to that diffused into the surrounding liquid medium. Since only the early-stage development of the nucleus growth is of interest, the set of equations is solved analytically by means of a small-time series expansion technique. A nonlinear Shanks transformation [13] is applied in order to resolve the convergence property of the series solution at larger time. Effect of surface tension on the temperature distribution in the liquid field and its influence on the growth rate of the interface are discussed. Also, to leadingorder approximation, the dependence of the phase growth rate on relevant physical parameters is established in an analytic form under the assumption of small degree of undercooling. These analytic functional relationships disclose important features pertaining to the underlying behavior of the problem that is otherwise unattainable by using the numerical computation technique.

\section{2}

\section{Formulation of the problem}

The unsteady heat conduction equation with spherical symmetry is:

$\frac{\partial T^{*}}{\partial t^{*}}=\alpha_{\ell}\left(\frac{2}{r^{*}} \frac{\partial T^{*}}{\partial r^{*}}+\frac{\partial^{2} T^{*}}{\partial r^{*^{2}}}\right), \quad a^{*} \leq R^{*}\left(t^{*}\right) \leq r^{*}<\infty$,

where $r^{*}$ is the spherical coordinate; $T^{*}$ is the temperature; $\alpha_{\ell}$ is the thermal diffusion coefficient of the liquid phase; $R^{*}\left(t^{*}\right)$ denotes the instantaneous location of the solid/liquid interface (see Fig. 1). Suppose that at $t^{*}=0^{+}$, a solid nucleus of radius $a^{*}\left(a^{*} \geq R_{0}^{*}\right)$ is suddenly introduced in a subcooled liquid whose temperature is initially kept at $T_{\infty}^{*}$ below the freezing point, then the proper boundary condition for all subsequent $t^{*}>0$ is

$T^{*} \rightarrow T_{\infty}^{*}$ as $r^{*} \rightarrow \infty$.

The equilibrium temperature at the interface is that given by the Gibbs-Thomson condition:

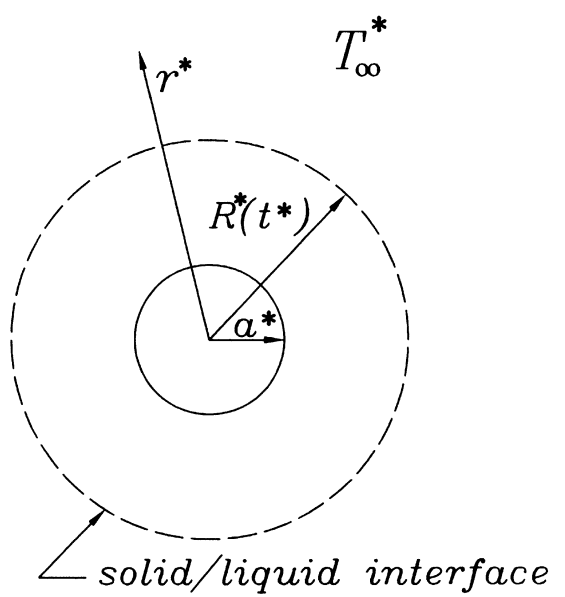

Fig. 1. Definition of physical domains of the problem
$T^{*}=T_{f}^{*}\left(1-\frac{\gamma}{L} \frac{2}{R^{*}\left(t^{*}\right)}\right) \quad$ at $r^{*}=R^{*}\left(t^{*}\right)$.

The equation for the motion of the solid/liquid interface is deduced from balancing the heat releasing rate and the heat conduction rate at the freezing front:

$L \frac{d R^{*}}{d t^{*}}=\left.\alpha_{\ell} c_{\ell} \frac{\partial T^{*}}{\partial r^{*}}\right|_{R^{*}}$,

where $c_{\ell}$ denotes the heat capacity (per unit volume) of the liquid phase.

Now, defining dimensionless variables as

$T=\frac{T^{*}-T_{f}^{*}}{T_{\infty}^{*}-T_{f}^{*}}, \quad r=\frac{r^{*}}{a^{*}}, \quad R=\frac{R^{*}}{a^{*}}, \quad t=\frac{\alpha_{\ell} t^{*}}{a^{*^{2}}}$,

the non-dimensional form of the governing equation (3) becomes:

$\frac{\partial T}{\partial t}=\frac{2}{r} \frac{\partial T}{\partial r}+\frac{\partial^{2} T}{\partial r^{2}}, \quad 1 \leq R(t) \leq r<\infty$.

The associated boundary conditions are

$T \rightarrow 1 \quad$ as $r \rightarrow \infty$

$T=\frac{\beta}{\Delta} \cdot \frac{1}{R(t)}=\frac{\sigma}{R(t)} \quad$ at $r=R(t)$,

and the non-dimensional growth rate of the freezing front is given by

$\frac{d R}{d t}=\left.\Delta \frac{\partial T}{\partial r}\right|_{R}$

In the above expressions, the dimensionless parameter $\Delta=c_{\ell}\left(T_{f}^{*}-T_{\infty}^{*}\right) / L$ is often called the Stefan number, which measures the degree of undercooling. The parameter $\beta=2 c_{\ell} \gamma T_{f}^{*} /\left(a^{*} L^{2}\right)$ represents the surface tension effect (Gibbs-Thomson effect), and $\sigma=\beta / \Delta$ is merely a convenient dimensionless parameter, which can also be taken as a measure of the surface tension when the Stefan number $\Delta$ is held fixed. Note that the admissible condition $a^{*} \geq R_{0}^{*}$ (see equation (2)) for a feasible phase growth imposes an upper limit on the parameter $\sigma$, i.e., $\sigma \leq 1$.

The problem involves a moving boundary (interface) whose position is part of the solution and hence is not known a priori. A boundary-fixed transformation [2]

$\eta=\frac{r-1}{R(t)-1}=\frac{r-1}{\tau(t)}$

is adopted first to map the unknown position of the freezing front to a fixed value in $\eta, \eta=1$. In equation (12), $\tau=R-1$ is another variable describing the instantaneous position of the freezing front, which can also be treated as a time-like coordinate $(0 \leq \tau<\infty)$. By using the chain rule, the original governing equation (8) can be rewritten in terms of the new coordinates $(\eta, \tau)$ 
$\frac{d R}{d t} \frac{\partial T}{\partial \tau}-\frac{\eta}{\tau} \frac{d R}{d t} \frac{\partial T}{\partial \eta}=\frac{2}{(1+\eta \tau) \tau} \frac{\partial T}{\partial \eta}+\frac{1}{\tau^{2}} \frac{\partial^{2} T}{\partial \eta^{2}}$.

A further transformation of temperature $T$ will place the equation in a form amenable to the series-expansion technique,

$$
T=\frac{\Theta}{r}=\frac{\Theta}{1+\eta \tau} \text {. }
$$

In terms of the new temperature variable $\Theta$, equation (13) becomes

$\tau^{2} \frac{d R}{d t} \frac{\partial \Theta}{\partial \tau}-\eta \tau \frac{d R}{d t} \frac{\partial \Theta}{\partial \eta}=\frac{\partial^{2} \Theta}{\partial \eta^{2}}$.

The corresponding boundary and interface conditions are

$\Theta \rightarrow 1+\eta \tau$ as $\eta \rightarrow \infty$,

$\Theta=\sigma \quad$ at interface $\eta=1$,

and the growth rate of the interface is governed by

$\frac{d R}{d t}=\frac{\Delta}{\tau}\left(\left.\frac{1}{1+\tau} \frac{\partial \Theta}{\partial \eta}\right|_{\eta=1}-\left.\frac{\tau}{(1+\tau)^{2}} \Theta\right|_{\eta=1}\right)$.

3

\section{Series solution}

The forms of equations (15) and (18) suggest that we can expand the solution into a power series of the time-like variable $\tau$, i.e.,

$\Theta(\eta, \tau)=\sum_{n=0} \tau^{n} \Theta_{n}(\eta)$

Substituting (19) into equations (15) and (18) and collecting the like-power terms in $\tau$, we obtain a system of equations for $\Theta_{n}$. The corresponding boundary conditions at each order $n$ are

$\Theta_{0}(\infty) \rightarrow 1, \quad \Theta_{1}(\infty) \rightarrow \eta ; \quad \Theta_{n}(\infty) \rightarrow 0 \quad$ for $n \geq 2$,

$\Theta_{0}(1)=\sigma, \quad \Theta_{n}(1)=0 \quad$ for $n \geq 1$.

Denoting $\lambda_{n}=\left.\frac{d \Theta_{n}}{d \eta}\right|_{\eta=1}$ the temperature gradient evaluated at the interface at each order $n$, the solution $\Theta_{n}$ to each different order equation is stated as follows.

\section{1}

$O\left(\tau^{0}\right)$

The equation at this order is

$\Theta_{0}^{\prime \prime}+\Delta \lambda_{0} \Theta_{0}^{\prime}=0$, where the prime symbols are shorthand notations for differentiations with respect to the coordinate $\eta$. The solution is easily obtained as

$$
\begin{aligned}
\Theta_{0} & =A_{0} \int_{\infty}^{\eta} e^{\frac{-\Delta \lambda_{0}}{2} \eta^{2}} d \eta+B_{0} \\
& =B_{0}-A_{0} \sqrt{\frac{\pi}{2 \Delta \lambda_{0}}}\left[1-\operatorname{erf}\left(\sqrt{\Delta \lambda_{0} / 2} \eta\right)\right]
\end{aligned}
$$

where $\operatorname{erf}(z)$ stands for the error function defined by

$\operatorname{erf}(z)=\frac{2}{\sqrt{\pi}} \int_{0}^{z} e^{-z^{2}} d z$

and

$A_{0}=\frac{(1-\sigma)}{\sqrt{\frac{\pi}{2 \Delta \lambda_{0}}}\left[1-\operatorname{erf}\left(\sqrt{\Delta \lambda_{0} / 2}\right)\right]}, \quad B_{0}=1$,

are the two integration constants determined from applying the boundary conditions (20) and (21). The temperature gradient at the interface is then obtained through differentiating equation (23):

$\lambda_{0}=\left.\frac{d \Theta_{0}}{d \eta}\right|_{\eta=1}=\frac{(1-\sigma) e^{\frac{-\Delta \lambda_{0}}{2}}}{\sqrt{\frac{\pi}{2 \Delta \lambda_{0}}}\left[1-\operatorname{erf}\left(\sqrt{\Delta \lambda_{0} / 2}\right)\right]}$.

Notice that equation (25) is an implicit function for the temperature gradient $\lambda_{0}$. For given $\Delta$ and $\sigma$, an iterative procedure is therefore required to calculate the numerical value of $\lambda_{0}$.

\section{2}

$O\left(\tau^{1}\right)$

The equation at this order is

$$
\begin{aligned}
\Theta_{1}^{\prime \prime}+\Delta \lambda_{0} \eta \Theta_{1}^{\prime}-\Delta \lambda_{0} \Theta_{1} & =-\Theta_{0}^{\prime \prime}-\Delta\left(\lambda_{1}-\sigma\right) \eta \Theta_{0}^{\prime} \\
& =\Delta A_{0}\left(\lambda_{0}-\lambda_{1}+\sigma\right) \eta e^{\frac{-\Delta i_{0}}{2} \eta^{2}} .
\end{aligned}
$$

The solution to this non-homogeneous equation is

$$
\begin{aligned}
\Theta_{1}= & \left.-A_{1}\left\{e^{\frac{-\Delta \lambda_{0}}{2} \eta^{2}}-\sqrt{\frac{\Delta \lambda_{0} \pi}{2} \eta} \eta 1-\operatorname{erf}\left(\sqrt{\Delta \lambda_{0} / 2} \eta\right)\right]\right\}+B_{1} \eta \\
& -\frac{A_{0}}{3 \lambda_{0}}\left(\lambda_{0}-\lambda_{1}+\sigma\right) \eta e^{\frac{-\Delta \lambda_{0}}{2} \eta^{2}}
\end{aligned}
$$

with integration constants

$$
A_{1}=\frac{1-\frac{A_{0}}{3 \lambda_{0}}\left(\lambda_{0}-\lambda_{1}+\sigma\right) e^{\frac{-\Delta \lambda_{0}}{2}}}{e^{\frac{-\Delta \lambda_{0}}{2}}-\sqrt{\frac{\Delta \lambda_{0} \pi}{2}}\left[1-\operatorname{erf}\left(\sqrt{\Delta \lambda_{0} / 2}\right)\right]}, \quad B_{1}=1 .
$$

At this order, the temperature gradient $\lambda_{1}$ at the interface is given by 


$$
\begin{aligned}
\lambda_{1}= & \left.\frac{d \Theta_{1}}{d \eta}\right|_{\eta=1}=\left\{1-\frac{\frac{A_{0}}{3 \lambda_{0}} e^{\frac{-\Delta \lambda_{0}}{2}} \sqrt{\frac{\Delta \lambda_{0} \pi}{2}}\left[1-\operatorname{erf}\left(\sqrt{\Delta \lambda_{0} / 2}\right)\right]}{e^{\frac{-\lambda_{0}}{2}}-\sqrt{\frac{\Delta \lambda_{0} \pi}{2}}\left[1-\operatorname{erf}\left(\sqrt{\Delta \lambda_{0} / 2}\right)\right]}-\frac{A_{0} e^{\frac{-\Delta \lambda_{0}}{2}}}{3 \lambda_{0}}\left(1-\Delta \lambda_{0}\right)\right\}^{-1} \\
& \times\left\{1+\frac{\sqrt{\frac{\Delta \lambda_{0} \pi}{2}}\left[1-\operatorname{erf}\left(\sqrt{\Delta \lambda_{0} / 2}\right)\right]\left[1-\frac{A_{0}}{3 \lambda_{0}}\left(\lambda_{0}+\sigma\right) e^{\frac{-\Delta \lambda_{0}}{2}}\right]}{e^{\frac{-\Delta \lambda_{0}}{2}}-\sqrt{\frac{\Delta \lambda_{0} \pi}{2}}\left[1-\operatorname{erf}\left(\sqrt{\Delta \lambda_{0} / 2}\right)\right]}-\frac{A_{0} e^{\frac{-\Delta \lambda_{0}}{2}}}{3 \lambda_{0}}\left(\lambda_{0}+\sigma\right)\left(1-\Delta \lambda_{0}\right)\right\} .
\end{aligned}
$$

In equations (28) and (29), it is seen that $\lambda_{1}$ and $A_{1}$ depend explicitly on $\lambda_{0}$ and $A_{0}$. Thus having determined the values of $\lambda_{0}$ and $A_{0}$ from the lowest order solution, all temperature gradients $\lambda_{n}$ and integration constants $A_{n}$ in subsequent higher-order solutions can be obtained explicitly in terms of theses fundamental values.

\section{3}

\section{$O\left(\tau^{2}\right)$}

The equation at this order is

$\Theta_{2}^{\prime \prime}+b \eta \Theta_{2}^{\prime}-2 b \Theta_{2}=\left(c-g \eta+h \eta^{3}\right) e^{\frac{-b}{2} \eta^{2}}$,

with

$b=\Delta \lambda_{0}, \quad c=\Delta A_{1}\left(\lambda_{0}-\lambda_{1}+\sigma\right), \quad g=\Delta A_{0}\left(\lambda_{0}-\lambda_{1}+\lambda_{2}+\sigma\right)$,

$h=\frac{A_{0}}{3} \Delta^{2}\left(\lambda_{0}-\lambda_{1}+\sigma\right)^{2}$.

The homogeneous part of equation (30) can be recast into a standard form via the coordinate transformation $\xi=$ $-b \eta^{2} / 2$ :

$\xi \Theta_{2}^{\prime \prime}+\left(\frac{1}{2}-\xi\right) \Theta_{2}^{\prime}+\Theta_{2}=0$.

Equation (32) belongs to the class of the confluent hypergeometric equation (Abramowitz and Stegun [14]), whose general form is

$\xi \Theta^{\prime \prime}+(q-\xi) \Theta^{\prime}-p \Theta=0$.

The two independent solutions to the above equation are

$M(p, q, \xi) \quad$ and $\quad \xi^{1-q} M(p-q+1,2-q, \xi)$

in which $M(p, q, \xi)$ is the confluent hypergeometric function of order $(p, q)$ defined as

$M(p, q, \xi)=\sum_{k=0}^{\infty} \frac{(p)_{k}}{(q)_{k}} \frac{\xi^{k}}{k !}$,

with

$$
\begin{aligned}
& (p)_{k}=p \cdot(p+1) \cdot(p+2) \cdots(p+k-1) \quad \text { for } k \geq 1, \\
& (q)_{k}=q \cdot(q+1) \cdot(q+2) \ldots(q+k-1) \quad \text { for } k \geq 1 \\
& (p)_{0}=1, \quad(q)_{0}=1 .
\end{aligned}
$$

Note that the power series in (35) terminates at finite number of terms for integer value of $p$. The present equation (32) corresponds to the case $(p, q)=(-1,1 / 2)$, and the two solutions are

$$
M\left(-1, \frac{1}{2}, \xi\right)=1-2 \xi=1+b \eta^{2} \quad \text { and } \quad \xi^{1 / 2} M\left(\frac{-1}{2}, \frac{3}{2}, \xi\right) .
$$

For certain special values of $(p, q)$, the hypergeometric function (35) can be related to the more familiar exponential and error functions [14], for examples:

$M(p, p, \xi)=e^{\xi}$ for any number $p$,

$M\left(\frac{1}{2}, \frac{3}{2}, \xi\right)=\frac{1}{\sqrt{-\xi}} \int_{0}^{\sqrt{-\xi}} e^{-z^{2}} d z=\frac{\sqrt{\pi}}{2 \sqrt{-\xi}} \operatorname{erf}(\sqrt{-\xi})$.

Thus by using the following relationships among hypergeometric functions of different orders ([14]):

$M(p, q, \xi)=M(p+1, q, \xi)-\frac{\xi}{q} M(p+1, q+1, \xi)$,

$(p-q) \xi M(p, q+1, \xi)=p q M(p+1, q, \xi)-q(p+\xi) M(p, q, \xi)$,

the second hypergeometric function in (36) is identified with

$$
\begin{aligned}
\xi^{1 / 2} M\left(\frac{-1}{2}, \frac{3}{2}, \xi\right) & =\frac{\xi^{1 / 2}}{2} M\left(\frac{3}{2}, \frac{3}{2}, \xi\right)+\xi^{1 / 2}\left(\frac{1}{2}-\xi\right) M\left(\frac{1}{2}, \frac{3}{2}, \xi\right) \\
& =\frac{1}{2} \sqrt{\frac{-b}{2}}\left[\eta e^{\frac{-b}{2} \eta^{2}}+\left(1+b \eta^{2}\right) \int_{0}^{\eta} e^{\frac{-b}{2} \eta^{2}} d \eta\right] .
\end{aligned}
$$

The two independent solutions to equation (32) can then be properly chosen as

$$
1+b \eta^{2} \text { and } \eta e^{\frac{-b}{2} \eta^{2}}-\sqrt{\frac{\pi}{2 b}}\left(1+b \eta^{2}\right)[1-\operatorname{erf}(\sqrt{b / 2} \eta)] \text {. }
$$

Having obtained the solution to the homogeneous part of equation (30), the particular solution can be found by using the method of variation of parameters. The complete solution for $\Theta_{2}$ is then

$$
\begin{aligned}
\Theta_{2}= & A_{2}\left\{\eta e^{\frac{-b}{2} \eta^{2}}-\sqrt{\frac{\pi}{2 b}}\left(1+b \eta^{2}\right)[1-\operatorname{erf}(\sqrt{b / 2} \eta)]\right\} \\
& +B_{2}\left(1+b \eta^{2}\right) \\
& +\frac{1}{8 b}\left(g-\frac{h}{b}\right) \sqrt{\frac{\pi}{2 b}}\left(1+b \eta^{2}\right)[1-\operatorname{erf}(\sqrt{b / 2} \eta)] \\
& -\left[\frac{c}{3 b}-\frac{1}{8 b}\left(g-\frac{h}{b}\right) \eta+\frac{h}{6 b} \eta^{3}\right] e^{\frac{-b}{2} \eta^{2}}
\end{aligned}
$$


with integration constants

$A_{2}=\frac{\left[\frac{c}{3 b}-\frac{1}{8 b}\left(g-\frac{h}{b}\right)+\frac{h}{6 b}\right] e^{\frac{-b}{2}}-\frac{(1+b)}{8 b}\left(g-\frac{h}{b}\right) \sqrt{\frac{\pi}{2 b}}[1-e r f(\sqrt{b / 2})]}{e^{\frac{-b}{2}}-(1+b) \sqrt{\frac{\pi}{2 b}}[1-e r f(\sqrt{b / 2})]}$,

$B_{2}=0$.

The temperature gradient $\lambda_{2}$ at this order is obtained from differentiating $\Theta_{2}$ in equation (37).

The homogeneous parts of the subsequent higher-order equations $(n \geq 2)$ can all be transformed into the standard form of a hypergeometric equation, procedures for acquiring their solutions are very similar to that stated above. In the present study, the solution has been determined up to $O\left(\tau^{5}\right)$. Since the expression of the solution becomes rather lengthy and cumbersome with increasing order $n$, results for higher order solutions will not be given here.

\section{4}

Results and discussion

\section{1}

\section{Temperature profiles}

Let us denote solutions of increasing order by a sequence $T_{m}$

$T_{m}=\frac{S_{m}}{1+\eta \tau}$,

where $S_{m}=\sum_{n=0}^{m} \tau^{n} \Theta_{n}$ is the first $m$-term partial sum of the series (19). In our present study, the value of $m$ is from 1 to 5 . Fixing the parameter values at $\Delta=0.1, \sigma=0.5$, Fig. 2 plots the temperature profiles $T_{m}$ of the first three orders at a time shortly after the initiation of the phase growth, $\tau=0.01$. It is seen that subsequent higher order solutions give consistent results at such a small time. However, for a larger value of $\tau, \tau=0.1$, the series solution diverges. As indicated in Fig. 3, the apparent large over- and undershoots appearing alternatively between the series sums of neighboring orders suggests that the power series has a nonphysical singularity at certain negative value of $\tau,{ }^{1}$ which severely limits the applicable range of the present result. One ingenious method to improve the convergence property of a slowly convergent or even divergent series is the nonlinear transformation proposed by Shanks [13]. According to Shanks, a new sequence $e_{m}^{(1)}$ is constructed from the original $T_{m}$ by the following transformation:

$e_{m}^{(1)}(T)=T_{m+1}-\frac{\left(T_{m+2}-T_{m+1}\right)\left(T_{m+1}-T_{m}\right)}{\left(T_{m+2}-T_{m+1}\right)-\left(T_{m+1}-T_{m}\right)}, \quad m \geq 1$.

Equation (40) is termed the first-order Shanks transformation. Similarly, the same transformation rule can be again applied on the newly formed sequence $e_{m}^{(1)}$ to yield a second new sequence $\boldsymbol{e}_{m}^{(2)}$ (second-order Shanks transformation):

\footnotetext{
${ }^{1}$ For example, the sign-alternating geometric series $1-\tau+\tau^{2}-\tau^{3}+\tau^{4}$ - ...., which is a correct expansion of the function $1 /(1+\tau)$, diverges for $\tau>1$ because $1 /(1+\tau)$ has a singularity at $\tau=-1$.
}

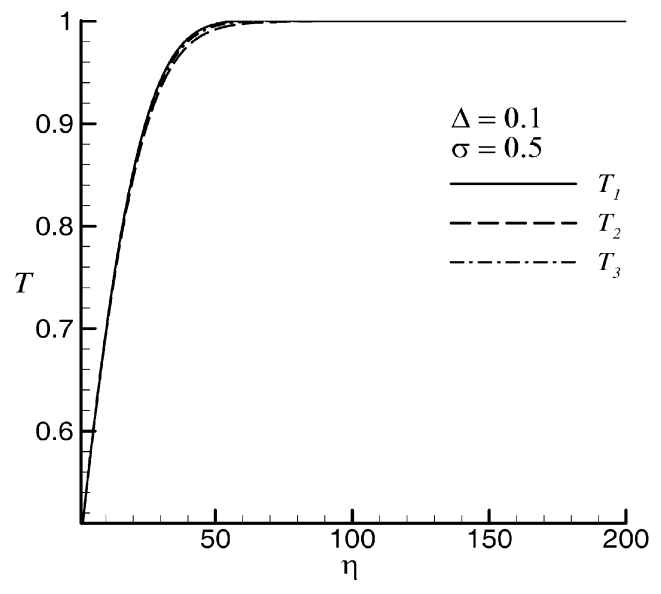

Fig. 2. First three orders solutions of the instantaneous temperature profile at $\tau=0.01$

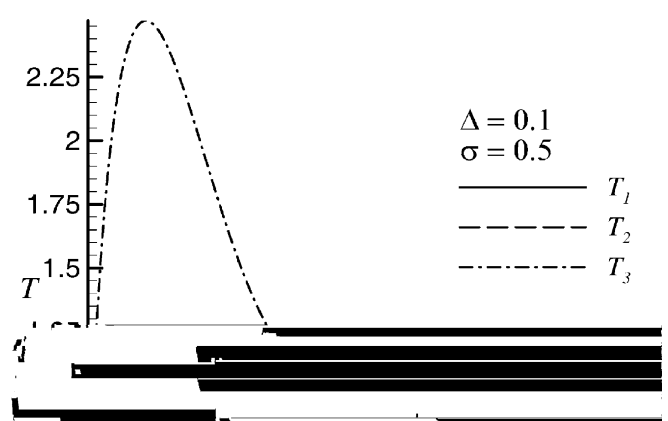

Fig. 3. First three orders solutions of the instantaneous temperature profile at $\tau=0.1$

$e_{m}^{(2)}(T)=e_{m+1}^{(1)}-\frac{\left(e_{m+2}^{(1)}-e_{m+1}^{(1)}\right)\left(e_{m+1}^{(1)}-e_{m}^{(1)}\right)}{\left(e_{m+2}^{(1)}-e_{m+1}^{(1)}\right)-\left(e_{m+1}^{(1)}-e_{m}^{(1)}\right)}, \quad m \geq 1$.

The process can be continued to produce still higher order Shanks transformation sequences. In the present case, the original temperature sequence $T_{m}$ is up to $m=5$ only; which allows for at most the employment of the secondorder Shanks transformation. Relationships between sequences generated by repeatedly applying the Shanks transformation are best described by the following triangular arrays:

$T_{1}$

$T_{2}$

$T_{3} e_{1}^{(1)}(T)$

$T_{4} e_{2}^{(1)}(T)$

$T_{5} e_{3}^{(1)}(T) e_{1}^{(2)}(T)$.

Shanks has demonstrated that the sequence formed by elements along the diagonal line has a much better conver- 
gence property than the original one. In cases when the original power series is 'geometric' or 'nearly geometric', the above Shanks transformation may even induce a converged sequence from the originally divergent one. ${ }^{2}$ Notion of employing the rational fraction (40) to approximate a series sum and the convergence property of the transformed diagonal sequence were discussed in details by Shanks [13]. Figures 4 and 5 provide at $\tau=0.1$ and 1.0 (which correspond to the instants when the solid nucleus grows to 1.1 and 2 times of its original size, respectively) the sequence of temperature profiles constructed from the diagonal elements $T_{1}, e_{1}^{(1)}(T)$, and $e_{1}^{(2)}(T)$ of the above triangular arrays. Clearly, the new sequence is now convergent under theses values of $\tau$. However, slight overshoot in the temperature profile is still observed in these figures, indicating that yet higher order Shanks transformation is needed.

Instantaneous temperature profiles at $\tau=0.01$ under different surface tension values $\sigma$ are plotted in Fig. 6 for $\Delta=0.1$. All temperature profiles shown in this plot are that from applying the second-order Shanks transformation, $e_{1}^{(2)}(T)$. It is seen that diffusion of the latent heat into the liquid field results in a thermal boundary layer adjacent to the interface. In reading this plot, it is reminded that in the definition of the dimensionless temperature $T$ (equation (7)), $T_{\infty}^{*}-T_{\mathrm{f}}^{*}$ is a negative quantity; thus the larger the value of the dimensionless temperature $T$ is, the lower is the physical temperature $T^{*}$. Figure 6 then indicates that the physical temperature at the interface is lower for larger surface tension $\sigma$ (as implied in the boundary condition (10)). Figure 6 also shows that the thickness of the thermal boundary layer increases with increasing surface tension. Because the difference between the temperature of the subcooled liquid and that at the surface of the nucleus becomes smaller for larger surface tension, the temperature gradient at the interface is reduced. Consequently, the growth rate of the solid nucleus is reduced (via equation (11)). Note that the temperature profiles in Fig. 6 are all plotted for the same value of $\tau(\tau=R-1)$. Since it takes longer time for a solid nucleus with larger interfacial surface tension to grow to the given size, the associated thermal boundary layer will thus diffuse a greater distance further into the liquid field.

\section{2}

\section{Growth rate of interface}

The growth rate of the solid nucleus is given by

$\frac{d R}{d t}=\frac{\Delta}{\tau}\left(\frac{\lambda_{0}+\tau \lambda_{1}+\tau^{2} \lambda_{2}+\tau^{3} \lambda_{3}+\tau^{4} \lambda_{4}+\tau^{5} \lambda_{5} \cdots}{1+\tau}-\frac{\tau \sigma}{(1+\tau)^{2}}\right)$.

As before, we may express increasingly higher order expansions of the phase growth rate by a sequence $R_{m}^{\prime}$ :

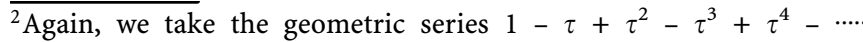
for example; the series diverges for $\tau>1$. However, applying Shanks transformation (40) to the first three partial sums of the above geometric series yields the 'exact' sum $1 /(1+\tau)$, regardless the value of $\tau$.
}

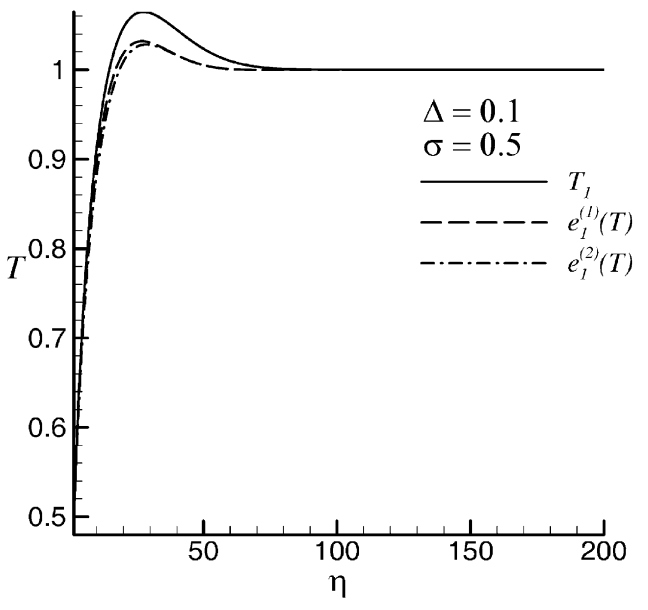

Fig. 4. Sequence of temperature profiles constructed from applying the first- and second-order Shanks transformations, $\tau=0.1$

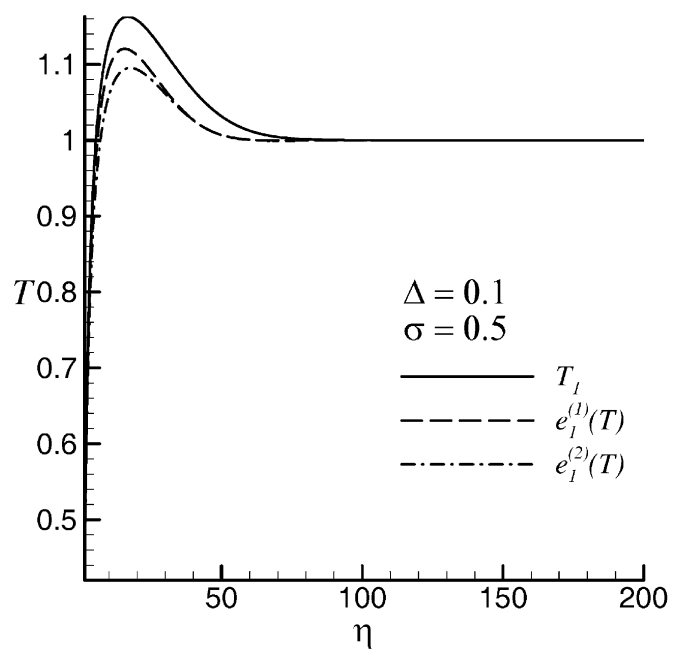

Fig. 5. Sequence of temperature profiles constructed from applying the first- and second-order Shanks transformations, $\tau=1$

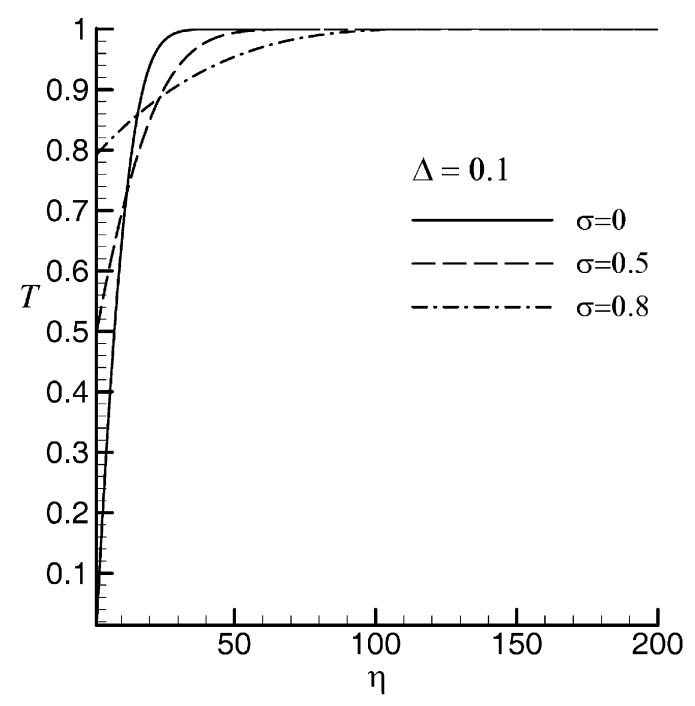

Fig. 6. Instantaneous temperature profiles at $\tau=0.01$ under different surface tension values 
$R_{m}^{\prime}=\frac{\Delta}{\tau}\left(\frac{S_{m}^{\prime}}{1+\tau}-\frac{\tau \sigma}{(1+\tau)^{2}}\right)$

where $S_{m}^{\prime}=\sum_{n=0}^{m} \tau^{n} \lambda_{n}$ denotes the first $m$-term partial sum of the temperature gradient $\lambda_{n}$ obtained from the series solution (19). Again, we may apply the first- and secondorder Shanks transformations to the above sequence $R_{m}^{\prime}$ to achieve converged results for large $\tau$. In analogy to (42), for example, we have the following triangular arrays constructed from the original sequence of the growth rate $R_{m}^{\prime}$ (which is evaluated at $\tau=1.0$ under $\Delta=0.005$ and $\sigma=0.5$ ), and sequences resulted from applying the first- and second-order Shanks transformations $e_{m}^{(1)}\left(R^{\prime}\right)$ and $e_{m}^{(2)}\left(R^{\prime}\right)$ :

$2.513 \times 10^{-3}$

$-1.919 \times 10^{-1}$

$2.780 \times 10^{2} \quad 2.377 \times 10^{-3}$

$-9.475 \times 10^{5}-1.103 \times 10^{-1}$

$1.390 \times 10^{18} \quad 2.780 \times 10^{2} \quad 2.331 \times 10^{-3}$.

Apparently, the original sequence $R_{m}^{\prime}$ diverges rather quickly with increasing order, whereas the diagonal sequence gives a converged result. It is also noticed that the first-order solution $R_{1}^{\prime}$

$R_{1}^{\prime}=\frac{\Delta}{\tau}\left[\frac{\lambda_{0}+\tau \lambda_{1}}{1+\tau}-\frac{\tau \sigma}{(1+\tau)^{2}}\right]$

already gives a value not too far from the converged result.

Figures 7 and 8 show the evolutions of the growth rate (after applying the second-order Shanks transformation, $\left.e_{1}^{(2)}\left(R^{\prime}\right)\right)$ under different surface tension values $\sigma$. Owing to the large temperature gradient across the initially very thin thermal boundary layer, the growth rate of the solid nucleus drops sharply from a theoretical value of infinity at the early stage of the solidifying process. As indicated by the figures, the value of the growth rate $d R / d t$ is greatly reduced when considering the surface tension effect at the interface. This is because increasing the surface tension reduces the tem-

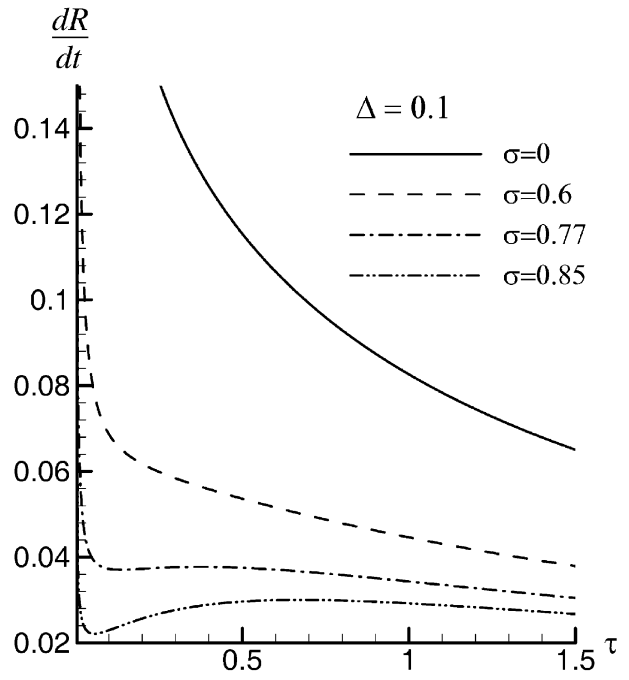

Fig. 7. Phase growth rate history under different surface tension values, $\Delta=0.1$

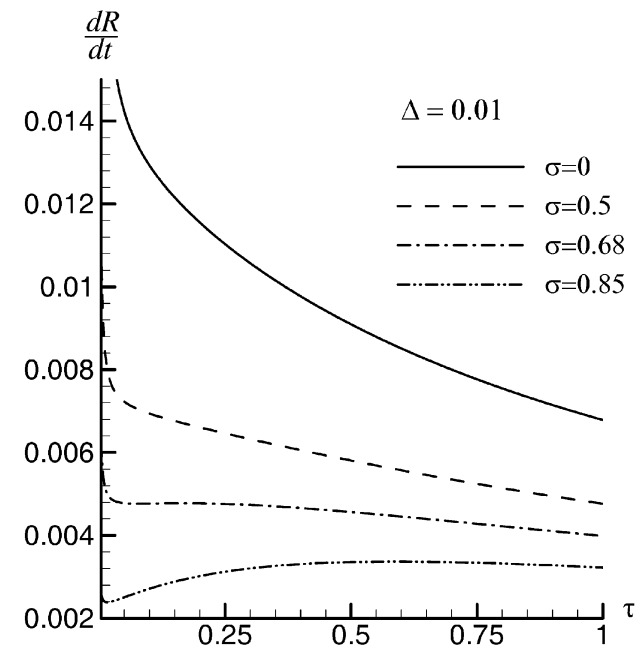

Fig. 8. Phase growth rate history under different surface tension values, $\Delta=0.01$

perature gradient at the interface (as stated in the previous section), and hence decreases the growth rate of the solid nucleus. It is also found that above certain critical value of surface tension $\sigma$, the decreasing of the growth rate becomes non-monotonic in $\tau$. Basically, the phase growth rate in this problem is governed by two mechanisms. Thickening of the solidified layer reduces the temperature gradient across the thermal boundary layer and hence reducing the growth rate of the interface, while the rising of the (dimensional) equilibrium temperature at the interface as the solid nucleus growing in size (according to the Gibbs-Thomson condition (10)) causes an increase in the temperature gradient at the interface and hence increasing the growth rate. The latter effect is more pronounced in the larger surface tension case. Competition of these two opposite mechanisms results in a critical time at which the growth rate of the solid nucleus reaches a local minimum, after that, the growth rate increases slowly with $\tau$. At still larger $\tau$ (and hence larger radius $R$ of the solid nucleus), the effect of surface tension becomes less significant (see equation (10)). The boundary-layer thickening effect dominates, and the phase growth rate starts to decrease again.

\section{3}

Approximate results of phase growth rate for $\Delta \ll 1$ The numerical example of the above Shanks triangular arrays (45) indicates that the first-order approximation

$\frac{d R}{d t} \approx R_{1}^{\prime}=\frac{\Delta}{\tau}\left[\frac{\lambda_{0}+\tau \lambda_{1}}{1+\tau}-\frac{\tau \sigma}{(1+\tau)^{2}}\right]$

still provides a satisfactory result for growth rate even at time as large as $\tau=1$. It is recalled that both $\lambda_{0}$ and $\lambda_{1}$ in (46) are functions of $\Delta$. If the Stefan number $\Delta$ is small (which is usually the case in many phase growth problems), we may derive the asymptotic expressions of $\lambda_{0}$ and $\lambda_{1}$ for $\Delta \ll 1$. After expanding the exponential and error functions at the right-hand-sides of equations (24), (25) and (29) for small $\Delta$, we have, correct to $O(\Delta)$, 
$\lambda_{0} \cong \frac{2 \Delta}{\pi}(1-\sigma)^{2}$

$A_{0} \cong \frac{2 \Delta(1-\sigma)^{2}}{\pi}$

$\lambda_{1} \cong \frac{3}{2}\left\{1-\frac{\sigma}{3}+\frac{3 \Delta}{2}(1-\sigma)\left(1-\frac{\sigma}{3}\right)-\frac{2 \Delta}{3 \pi}(1-\sigma)^{2}\right\}$.

On substituting (47)-(49) into equation (46), the analytical approximation of the growth rate is obtained as

$$
\begin{aligned}
\frac{d R}{d t} \cong & \frac{\Delta}{\tau}\left\{\frac{2 \Delta}{\pi(1+\tau)}(1-\sigma)^{2}+\frac{3 \tau}{2(1+\tau)}\left[\left(1-\frac{\sigma}{3}\right) .\right.\right. \\
& \left.\left.+\frac{3 \Delta}{2}(1-\sigma)\left(1-\frac{\sigma}{3}\right)-\frac{2 \Delta}{3 \pi}(1-\sigma)^{2}\right]-\frac{\tau \sigma}{(1+\tau)^{2}}\right\} .
\end{aligned}
$$

Depending on the relative orders of magnitude of the parameters $\Delta$ and $\tau$, we may further distinguish the following three cases:

(i) $\tau \ll \Delta \ll 1$ (approximation 1 )

In this parameter range, equation (50) is dominated by the term

$$
\frac{d R}{d t} \cong \frac{2 \Delta^{2}}{\tau \pi}(1-\sigma)^{2}
$$

(ii) $\tau \approx \Delta \ll 1$ (approximation 2 )

In this parameter range, the leading-order terms in equation (50) are

$$
\frac{d R}{d t} \cong \frac{\Delta}{\tau}\left\{\frac{2 \Delta(1-\sigma)^{2}}{\pi}+\frac{3 \tau(1-\sigma)}{2}\right\}
$$

(iii) $\Delta \ll 1 \approx \tau$ (approximation 3 )

In this parameter range, the leading-order approximation of equation (50) is

$$
\frac{d R}{d t} \cong \frac{3 \Delta}{2(1+\tau)^{2}}\left\{1-\sigma+\tau\left(1-\frac{\sigma}{3}\right)\right\} .
$$

Equations (51)-(53) provide in each different range of time $\tau$ the leading-order approximations of the phase growth rate in terms of relevant physical parameters $\Delta, \sigma$ and $\tau$ under the condition of small Stefan number $\Delta \ll 1$. Combining the above approximations, we may derive a general expression (designated as approximation 4) valid for all ranges of $\tau$ up to $\tau \sim O(1)$ :

$\frac{d R}{d t} \cong \frac{\Delta}{\tau}\left\{\frac{2 \Delta}{\pi(1+\tau)}(1-\sigma)^{2}+\frac{3 \tau}{2(1+\tau)}\left(1-\frac{\sigma}{3}\right)-\frac{\tau \sigma}{(1+\tau)^{2}}\right\}$.

The four expressions are plotted in Fig. 9 for $\Delta=0.01$, $\sigma=0.85$. In each plot, more accurate result from the second-order Shanks transformation $e_{1}^{(2)}\left(R^{\prime}\right)$ of the original sequence $R_{m}^{\prime}$ (with numerically evaluated $\lambda_{n}$ ) is
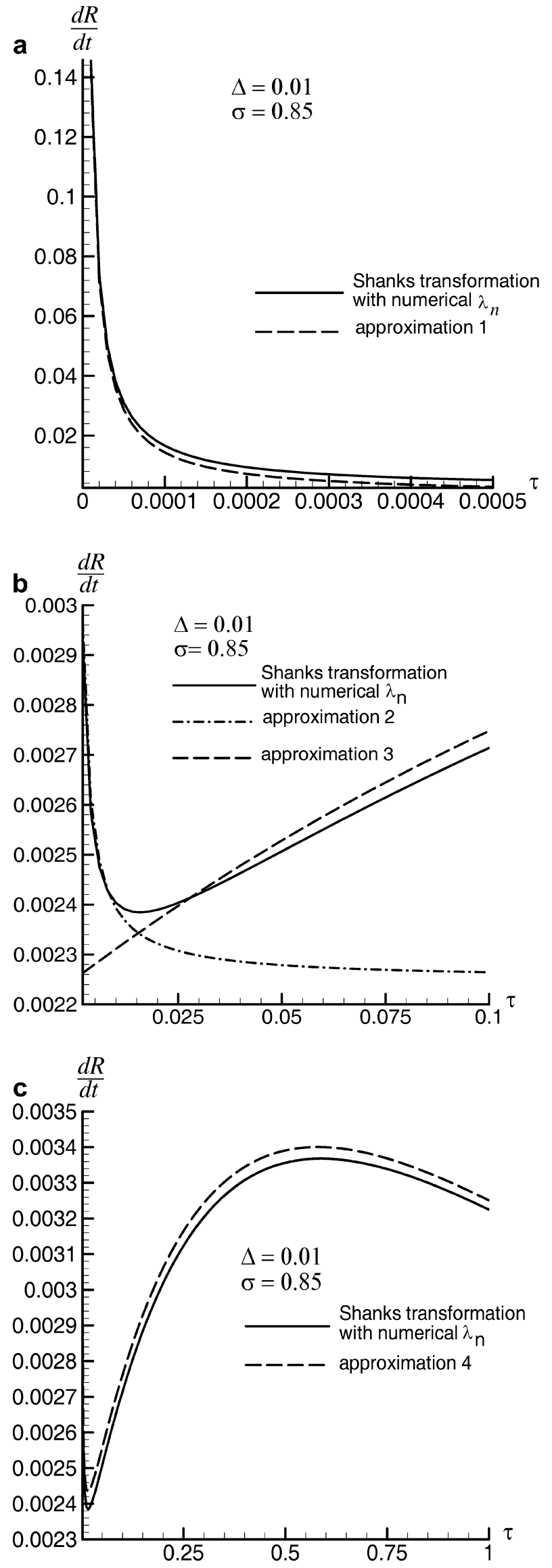
Fig. 9. Comparisons between the asymptotic results of the phase growth rate and that obtained from the original series solution (with Shanks transformation) in the parameters ranges (a) $\tau \ll \Delta \ll 1$, (b) $\tau \approx \Delta \ll 1$ and $\Delta \ll 1 \approx \tau$ (c) all $\tau$ up to $\tau \sim O(1)$

also provided for comparison. Clearly, both results are in close agreement. The largest discrepancy in approximation 4 is around 2\% only. Expressions (51)-(53) allow us to assess explicitly the effect of the surface tension on the growth rate of the interface at different stages of its development. For instance, at small $\tau$ the growth rate falls off quadratically with increasing surface tension; while the variation becomes linear when $\tau$ reaches $O(1)$.

\section{4}

\section{Critical surface tension}

It has been mentioned previously that the decreasing of the phase growth rate becomes non-monotonic with $\tau$ when the surface tension $\sigma$ is above certain critical value. Such critical surface tension value can be determined readily if one adopts the approximate form of the growth rate, equation (46). The criterion for local extremes to occur in the growth rate is

$$
\begin{aligned}
& \frac{d}{d \tau}\left(\frac{d R}{d t}\right) \\
& =\frac{\Delta}{\tau^{2}(1+\tau)^{3}}\left\{-\lambda_{0}(1+\tau)(1+2 \tau)-\lambda_{1} \tau^{2}(1+\tau)+2 \sigma \tau^{2}\right\}=0,
\end{aligned}
$$

which results in the finding of the roots to the following cubic equation

$$
\tau^{3}+\left(1+\frac{2 \lambda_{0}}{\lambda_{1}}-\frac{2 \sigma}{\lambda_{1}}\right) \tau^{2}+\frac{3 \lambda_{0}}{\lambda_{1}} \tau+\frac{\lambda_{0}}{\lambda_{1}}=0
$$

The situation that a local minimum-maximum pair is just about to form in the growth rate history is equivalent to the requirement that the above cubic equation has a repeated root. The condition under which the cubic equation (55) exhibits a double-root can be deduced from a standard polynomial analysis (see, e.g. [15]), and this leads to a function relationship among parameters

$f\left(\sigma, \lambda_{0}, \lambda_{1}\right)=0$.

Recalling that both $\lambda_{0}$ and $\lambda_{1}$ are functions of $\Delta$ and $\sigma$, the above relationship then gives implicitly the critical value $\sigma$ as a function of $\Delta$.

Equation (56) has been solved numerically for $\sigma$ under various given values of Stefan number $\Delta$, the result is plotted in Fig. 10. The critical $\sigma$ increases only slightly from 0.64 to 0.76 as the Stefan number $\Delta$ ranges from 0.001 to 0.1 . However, it is reminded that the true surface tension parameter is $\beta=\Delta \sigma$; thus the critical surface tension value actually increases drastically with the Stefan number.

\section{5}

\section{Concluding remarks}

In this paper, the thermal effect of the interfacial surface tension on the early-stage phase growth of a spherical solid

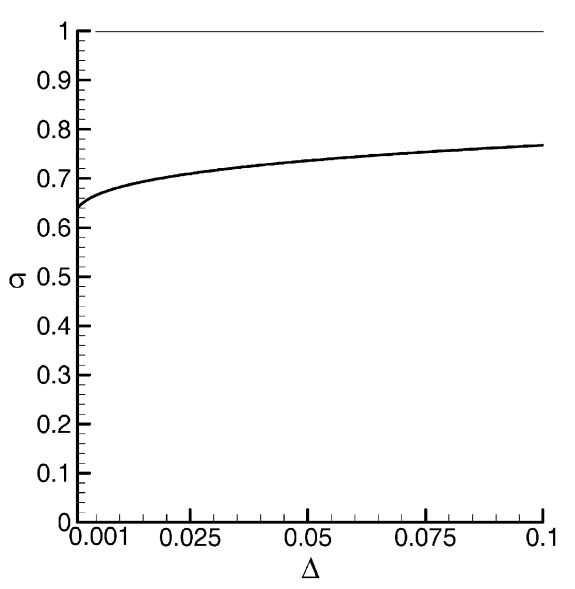

Fig. 10. Critical surface tension $\sigma$ as a function of Stefan number $\Delta$. The horizontal line $\sigma=1$ is the upper limit value of the parameter $\sigma$ above which solidification is not feasible. See explanation in the introduction section

nucleus immersed in a subcooled medium has been investigated analytically by using a small-time series expansion technique. A nonlinear Shanks transformation has been applied to the series solution as an attempt to extend its applicability to a larger time range. In summary, the present study shows that surface tension reduces the equilibrium temperature at the solid/liquid interface and hence reduces the growth rate of the interface. In the vanishing surface tension case, the phase growth rate drops monotonically with time as predicted. However, new feature has been disclosed when considering the surface tension effect. Existence of a critical surface tension has been found above which the decreasing of the phase growth rate becomes non-monotonic. The leading-order expression of the phase growth rate in each different time range has also been derived analytically under the condition of small Stefan number. The results indicate that increasing the surface tension causes a quadratic reduction in the phase growth rate at small time and a linear reduction at later time.

In real phase growth problems, the solid crystal is seldom of spherical in geometry. Even if the configuration is truly spherical at the incipience of solidification, the nucleus cannot preserve its radial symmetry, due to instability, when it grows beyond certain critical size. This critical radius is usually small (about $7 R_{0}^{*}$ from the linear stability analysis of [4]), thus the study conducted in this paper is somewhat restricted in practical applications. Nevertheless, the present work provides a fully nonsteady result for the simplest possible phase growth model which represents one of the few cases that can be treated by theoretical analysis. It is anticipated that the physical insight we gain from this simple example should assist us in formulating a more realistic local evolution model $([8,16])$ for interfacial growth as a tractable simplification of the extremely complex dynamics of pattern formation.

\section{References}

1. Rubinstein LI (1971) The Stefan problem. Translations of Mathematical Monographs 27: Am Math Soc 
2. Hill JM (1987) One-dimensional Stefan problems. Essex: Longman Scientific and Technical

3. Frank FC (1950) Radially symmetric phase growth controlled by diffusion. Proc Roy Soc A 201: 586-599

4. Mullins WW; Sekerka RF (1963) Morphological stability of a particle growing by diffusion or heat flow. J Appl Phys 34: 323-329

5. Langer JS; Muller-Krumbhaar H (1978) Theory of dendritic growth - I. Elements of a stability analysis. Acta Metall 26: 16811687

6. Langer JS (1980) Instabilities and pattern formation in crystal growth. Rev Mod Phys 52: 1-28

7. Xu JJ (1990) Asymptotic theory of steady axisymmetrical needlelike crystal growth. Stud Appl Math 82: 71-91

674 8. Kessler DA; Koplik J; Levine H (1985) Geometrical models of interface evolution, III. Theory of dendritic growth. Phys Rev A 31: 1712-1717

9. Caroli B; Caroli C; Roulet B; Langer JS (1986) Solvability condition for needle crystals at large undercooling in a nonlocal model of solidification. Phys Rev A 33: 442-452
10. Amar MB; Moussallam B (1987) Numerical results on twodimensional dendritic solidification. Physica 25D: 155-164

11. Turnbull D (1956) Phase changes. In: Solid state physics (Seitz F; Turnbull D eds.) 3 New York: Academic Press

12. Hurle DTJ ed. (1993) Handbook of crystal growth, volume I: Fundamentals. Amsterdam: North-Holland

13. Shanks D (1955) Nonlinear transformations of divergent and slowly convergent sequences. J Math Phys 34: 1-42

14. Abramowitz M; Stegun IA eds. (1965) Handbook of mathematical functions. New York: Dover

15. Press WH; Teukolsky SA; Vetterling WT; Flannery BP (1992) Numerical recipes. 2nd edn. Cambridge: Cambridge University Press

16. Brower RC; Kessler DA; Koplik J; Levine H (1984) Geometrical models of interface evolution. Phys Rev A 29: 1335-1342 